\title{
Control Of Process Variables For Remote Applications Using Lab VIEW And Internet
}

\author{
S.Harivardhagini ${ }^{1}$ and S.Pranavanand ${ }^{2}$ \\ ${ }^{1}$ CVR College of Engineering, Department of EIE., Ibrahimpatan, R.R.District, A.P., India \\ Email:harivardini@gmail.com \\ ${ }^{2}$ VNR Vignana Jyothi Institute of Engineering \& Technology, R.R.District, A.P., India \\ Email:pranavanand_s@vnrvjiet.in
}

\begin{abstract}
The paper presents a system that monitors and controls process variables for remote applications. LabVIEW (short for Laboratory Virtual Instrumentation Engineering Workbench) is a visual programming language commonly used for Data Acquisition (DAQ) and control. LabVIEW contains a user interface, known as front panel objects. By this we can access the acquired process variables over the internet. The accessed process variables like temperature, pressure, strain or any other can be suitably conditioned and feedback for control of the process via the internet for control. The system would find wide application in processes which are hazardous in nature. People can be safeguarded from hazardous effect, in dangerous areas like chlorine industry or at places where person gets affected by dangerous gases. This note presents one such idea using LabVIEW and Internet.
\end{abstract}

Index Terms-LabVIEW, Process variables, PID control Graphical system design, strain measurement, compact field point.

\section{INTRODUCTION}

Modern process variable measurement (level, temperature, strain) is truly sophisticated. Standard laboratories around the world routinely measure process variables (temperature, strain, energy, etc.,) to one thousand of a unit. This can even be done automatically with computer controlled equipment. The methods of process variable measurement have changed rapidly in recent years. Inexpensive commercial equipment for measurement and control often will indicate measurement to one tenth of a unit. Because of adverse environments, sensors or instrument instability, vibration, electrical noise, or design comprises, the instruments may have errors whose magnitude may vary over a large scale. It is therefore required to understand the entire process before any valuable measurement is recorded.

The concept of measurement of process variables like level, temperature and strain might have originated long back and even still innovation is on for this field of science.

In physics, temperature is a physical property of a system that underlines the common notions of hot and cold. Temperature is one of the principal parameters of thermo dynamics. On the macroscopic scale, temperature is the unique physical property that determines the direction of heat flow between two objects placed in thermal contact. If no heat flow occurs, the two objects have the same temperature; otherwise heat flows from the hotter object to the colder object. On the microscopic scale, temperature can be defined as the average energy in each degree of freedom in the particles in a system. For a solid, this energy is found in the vibrations of its atoms about their equilibrium positions.

The level can be measured using capacitive transducer. The capacitive transducer or sensor is nothing but the capacitor with variable capacitance. The capacitive transducer comprises of two parallel metal plates that are separated by the material such as air, which is called as the dielectric material. In the typical capacitor the distance between the two plates is fixed, but in variable capacitance transducers the distance between the two plates is variable. In the instruments using capacitance transducers the value of the capacitance changes due to change in the value of the input quantity that is to be measured. This change in capacitance can be measured easily and it is calibrated against the input quantity, thus the value if the input quantity can be measured directly.

The strain is measured using strain gauge. The strain gauge has been in use for many years and is the fundamental sensing element for many types of sensors, including pressure sensors, load cells, torque sensors, position sensors, etc. The majority of strain gauges are foil types, available in a wide choice of shapes and sizes to suit a variety of applications. They consist of a pattern of resistive foil which is mounted on a backing material. They operate on the principle that as the foil is subjected to stress, the resistance of the foil changes in a defined way. The strain gauge is connected into a Wheatstone bridge circuit with a combination of four active gauges (full bridge), two gauges (half bridge), or, less commonly, a single gauge (quarter bridge). In the half and quarter circuits, the bridge is completed with precision resistors. If a strip of conductive metal is 
stretched, it will become skinnier and longer, both changes resulting in an increase of electrical resistance end-to-end. Conversely, if a strip of conductive metal is placed under compressive force (without buckling), it will broaden and shorten. If these stresses are kept within the elastic limit of the metal strip (so that the strip does not permanently deform), the strip can be used as a measuring element for physical force, the amount of applied force inferred from measuring its resistance. Such a device is called a strain gauge. Modern process variable measurement is truly sophisticated.

The paper is organized as follows .Section I Introduction to measuring system, techniques used for measurement of process variables considered in our process. Section II: Block diagram of the process considered, Section III: Overview of compact field point, Section IV: The Virtual Instrument, Section V: Results, Section VI: Conclusion and future enhancement, Section VII: Acknowledgement, Section VIII references.

\section{Block Diagram Of The Process}

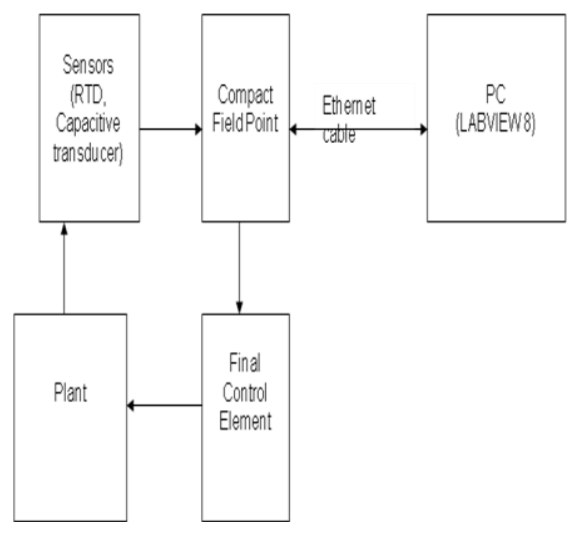

Figure 1. Block Diagram

The aim of this process is to get the online values of the process variables, providing them to control terminal and finally actuating a required control action. Communication in this particular process is done through Ethernet so that the control action/monitoring can be done from anywhere in the world if the required authentication is successful.

\section{OVER VIEW OF CFP}

Compact field point is an easy-to-use, highly expandable programmable automation controller (PAC) composed of rugged I/O modules and intelligent communication interfaces. The compact field point I/O modules filter, calibrate, and scale raw sensor signals to engineering units, as well as perform self-diagnostics to look for problems, such as open thermocouple. Through its built-in web and file servers, the compact field point interface automatically publishes measurements over the Ethernet network. Plus, connect to virtually any sensor type with the wide variety of I/O modules, such as thermocouples, RTDs, strain gages, $4-20 \mathrm{~mA}$ sensors, and a variety of digital signals from 5-30 VDC and 0250 VAC. It can Deploy real-time embedded controllers for stand-alone data logging, advanced analysis, and process control. It can access I/O points nearby or miles away on the network using the same simple read and write software framework. The compact field point can connect virtually any sensor directly to the wide variety of high-accuracy analog and discrete I/O modules. It has LabVIEW real-time embedded controllers for control, measurement, and signal processing. It can operate as stand-alone embedded real-time controllers or PC- based distributed I/O Ethernet interface

\section{The ViRTUAL INSTRUMENT}

Virtual instrument is the code/program developed using LabVIEW. They are also referred as Graphical System Design (GSD). It has two parts namely Front Panel and Block diagram. The former gives the look of any traditional instrument on the computer screen whereas the latter provides a platform to write our logic. This also enables is create some check points with the loss of programs performance. It is extremely user friendly and no prerequisite programming skills is required.

The Virtual Instrument offers many tools for easy debugging. LabVIEW offers a variety of tools kits required for various domains staring from basic measurement to high end controller design, signal processing to high speed rocket trajectory control, image processing to biomedical engineering. 
The following are the block diagram and front panel of level process control.
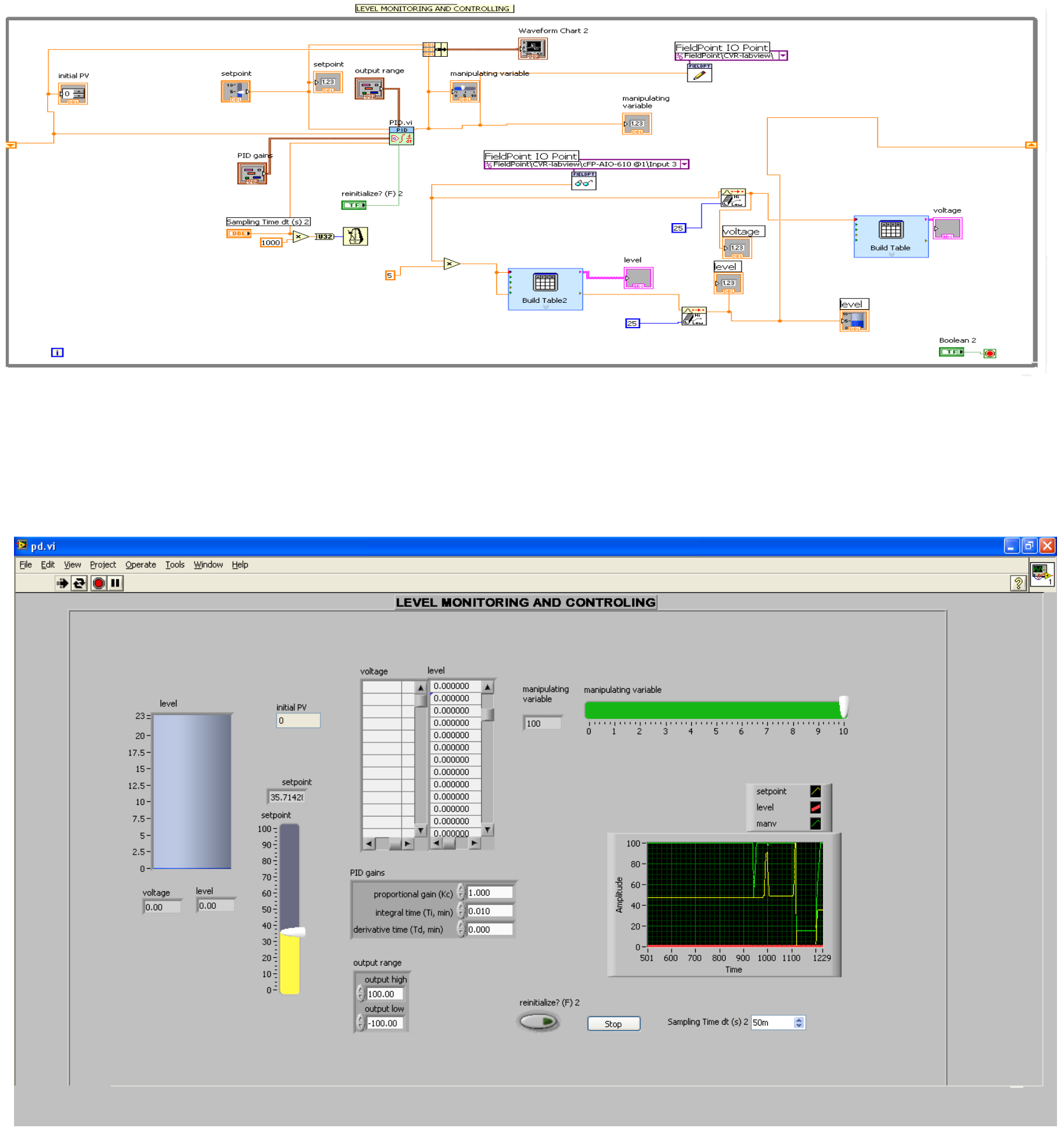

Figure 3. Front panel of the level monitoring and control 
The following are the block diagram panel and front panel of strain monitoring process.
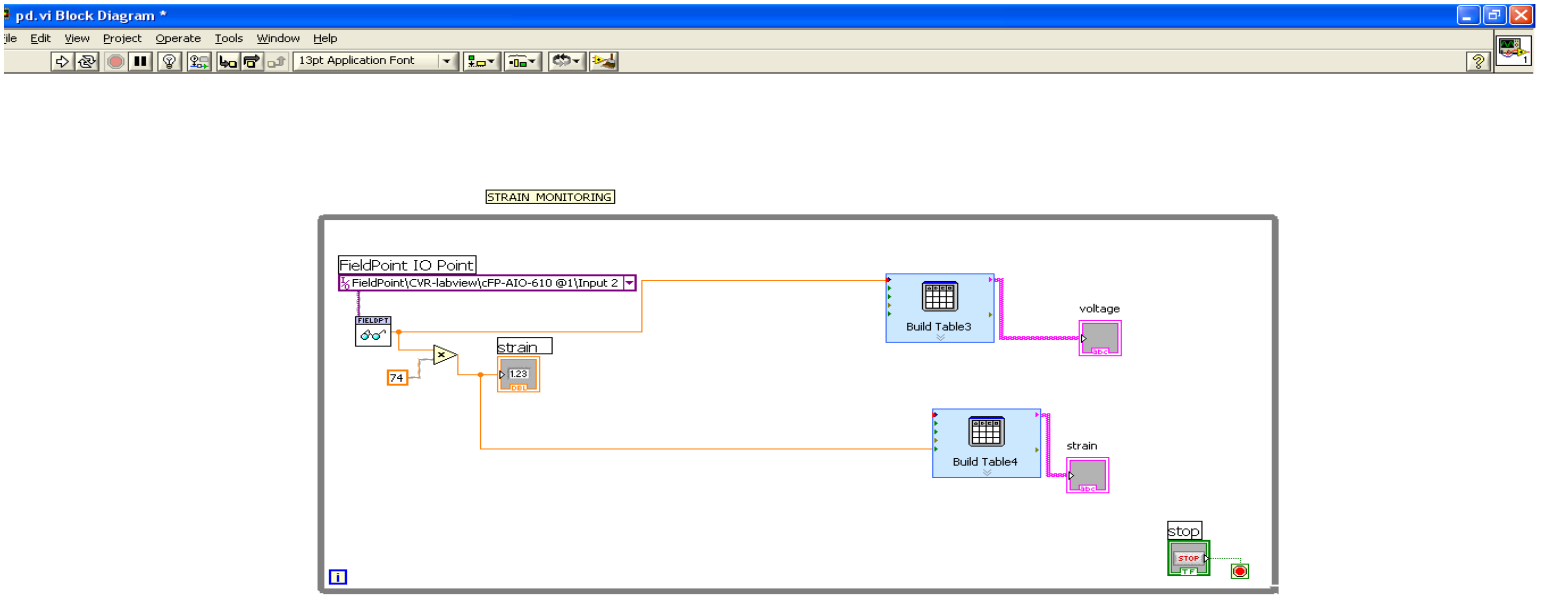

Figure 4. Block Diagram of the strain monitoring and control

The strain applied to a cantilever beam is measured and monitored through internet.

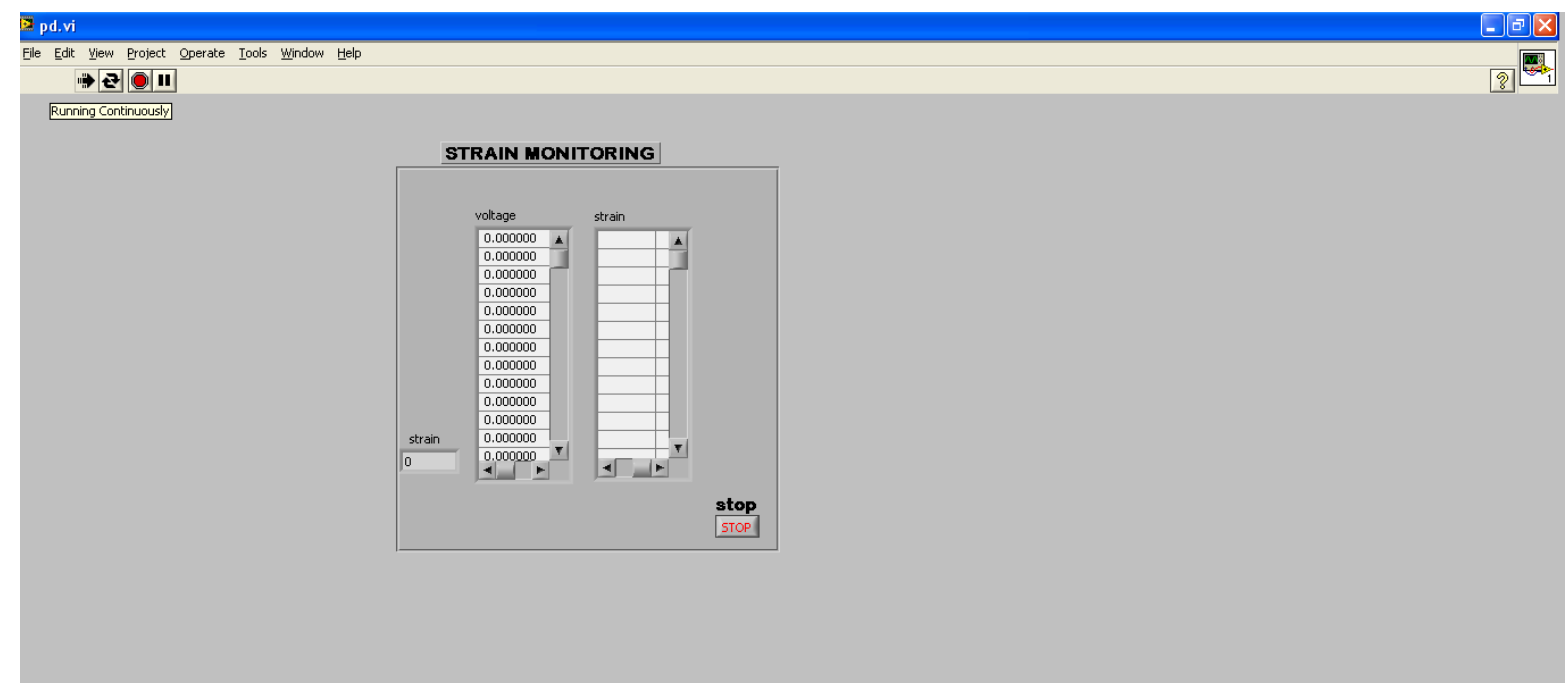

Figure 5. Front panel of the strain monitoring 
The temperature measurement and control of a water bath is done. The sensor used is RTD (Pt 100). The following are the block diagram panel and front panel of temperature monitoring and control process.

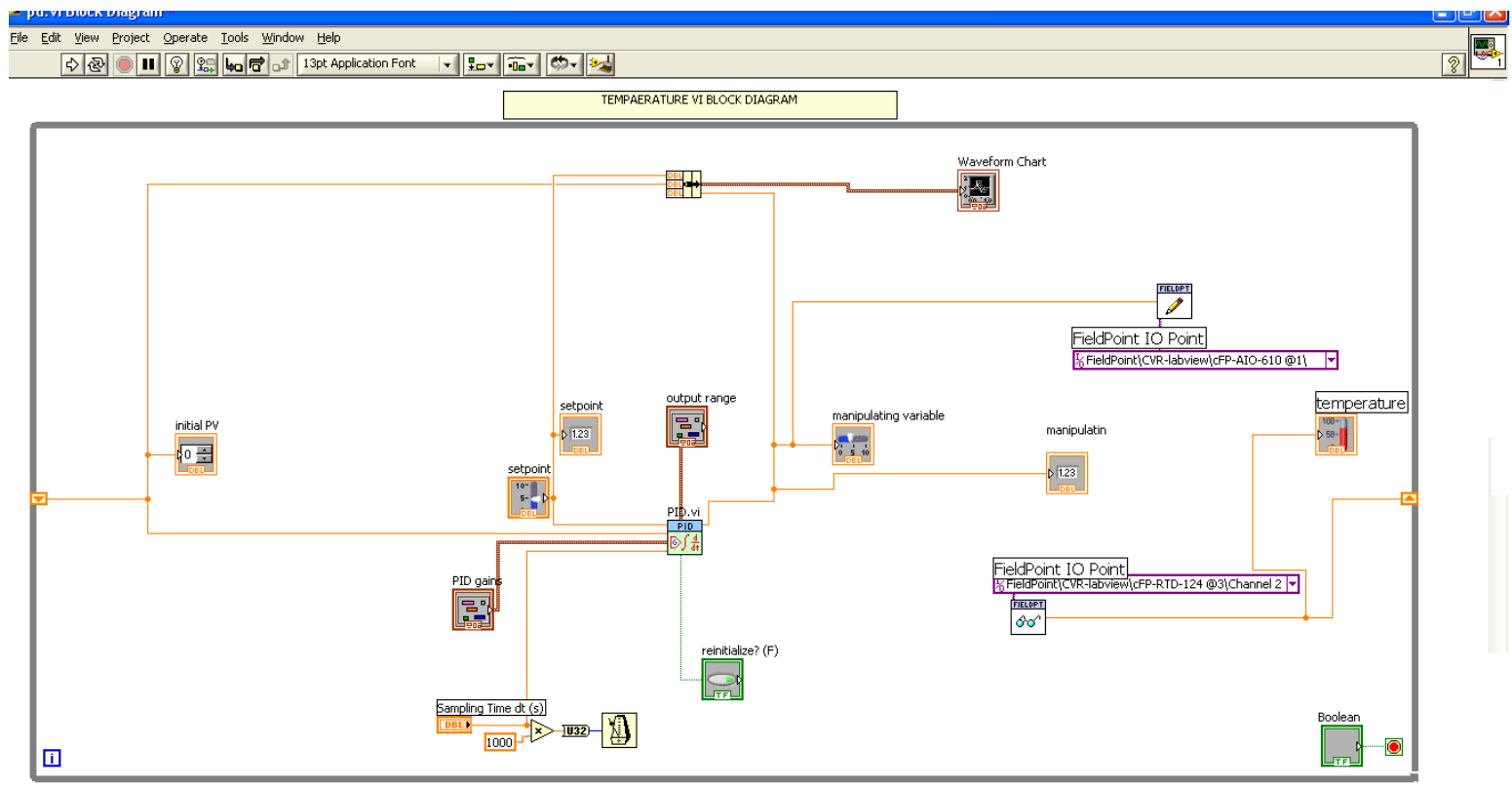

Figure 6. Block diagram of the temperature monitoring and control

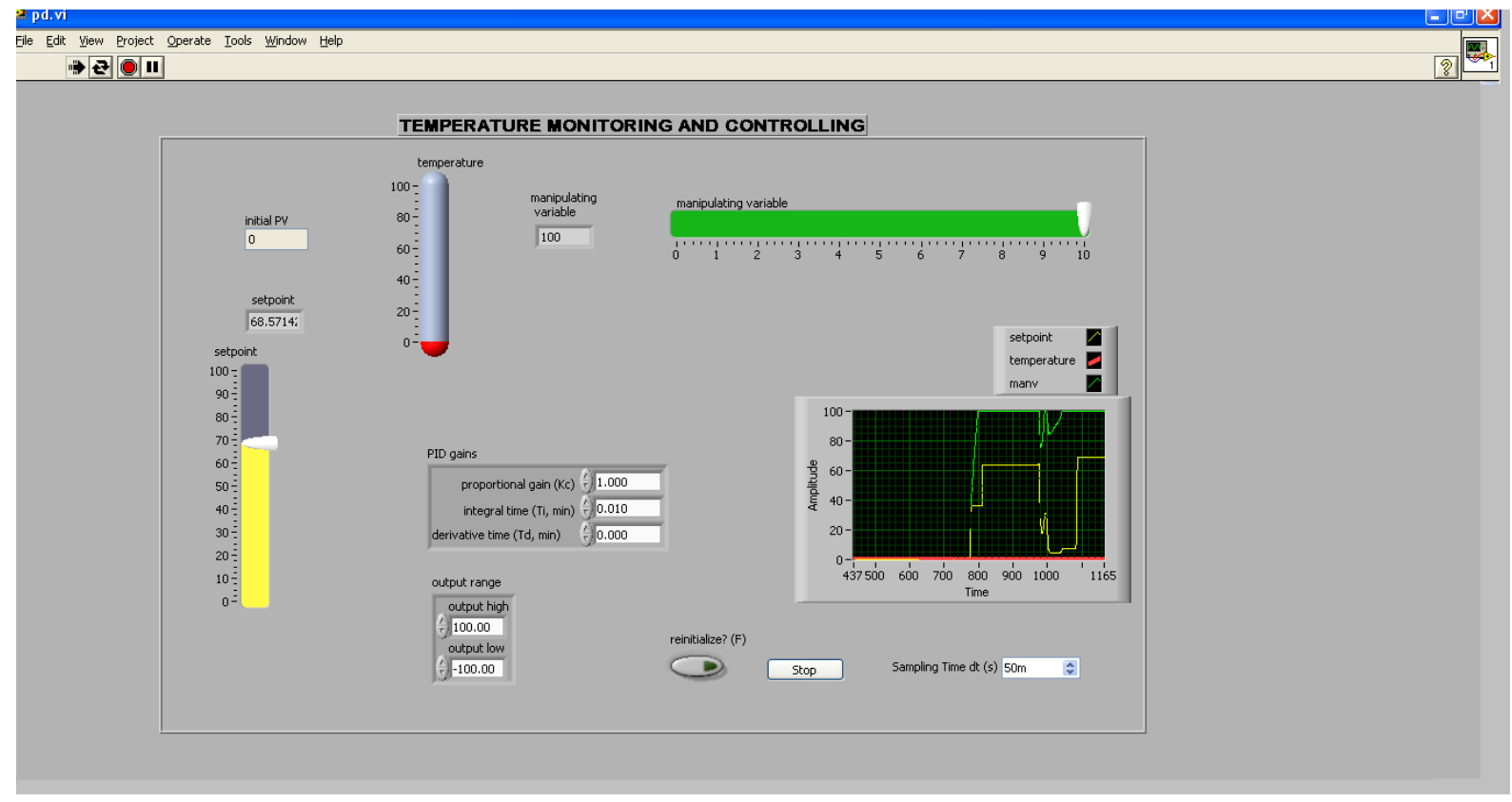

Figure 7. Front panel of the temperature monitoring and control 
The results are shown below. The level and temperature of kerosene tank and water bath respectively were monitored and controlled through internet. Strain of cantilever beam was monitored through internet.

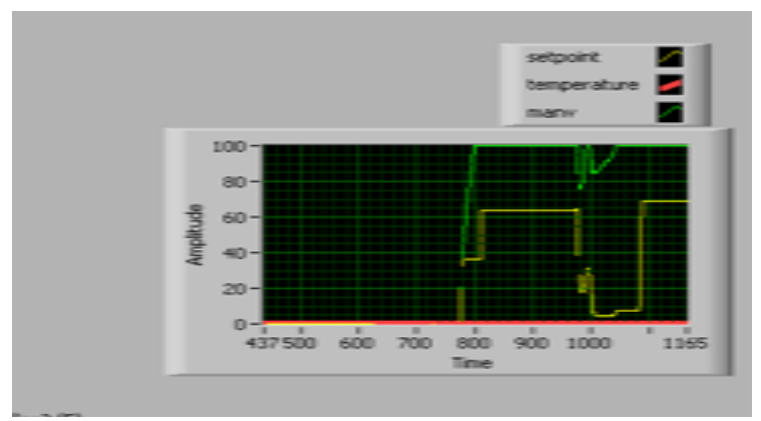

Figure 8.Graph of the level monitoring and control using PID control

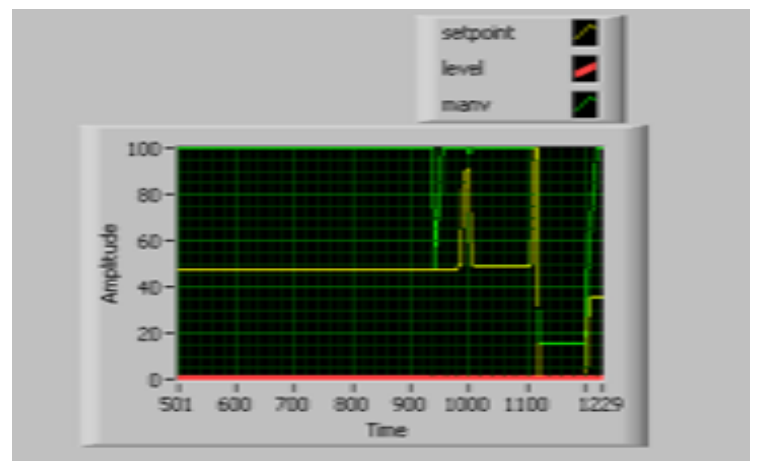

Figure 9.Graph of the temperature monitoring and control using PID control

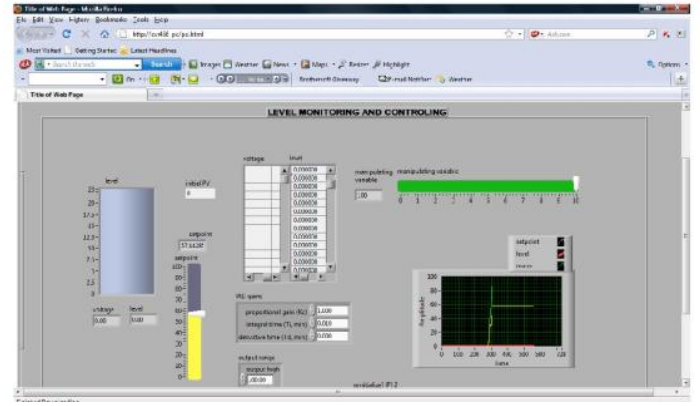

Figure 10. Internet Access

\section{CONCLUSION AND FutuRE ENHANCEMENT}

This paper deals with the process variable acquisition and control of level, temperature and also monitoring of process variable like level, temperature and strain through internet using Lab View. This is something which is different from the traditional practice. The general procedure is to dump the PID control algorithm into a microcontroller, now anyone adept at microcontroller programming will not agree with us that, the program runs into pages and more. So if the process is a real time control loop. The same task has been achieved by us within a single page.

The controller's output which we have obtained is to be signal conditioned to match the voltage level's of the final control element, SCR (silicon controlled rectifier in our case), therefore there still exists some complexity and thought process to be put into. So as a small scale demonstration we have shown the control using an LED at the output. The intensity of the LED output light varied as the PID control also varied.

The process of tuning the controller used by us is not completely scientific and therefore we would recommend an improvement in that part. There exists a myriad of ways which can be implemented viz. Z-N method, PRC method etc.

We would like to conclude by leaving it to the readers to ponder over the utility of the above project reminding them that the process can be a part of a larger project which might include more number of parameters to be controlled and the system being more complex.

\section{ACKNOWLEDGEMENT}

We thank the authorities of C.V.R. College of Engineering for providing support for this research work.

\section{REFERENCES}

[1] Barry E. Paton 1999," LabVIEW Graphical Programming for Instrumentation". Prentice Hall PTP, New Jersey, U.S.A.

[2] Curtis D. Johnson 1984," Microprocessor-Based Process Control". Prentice-Hall International, Inc Englewood Cliffs, NJ, U.S.A.

[3] DE-LORENZO, 2002, Electronic Laboratory," (Basic Board To Study Temperature Regulation)".DL.2155RGT1, Dl.2155RGT2, Milano, Italy.

[4] Donald R. Coughanowr, 1991,"Process Systems Analysis and Control". McGraw-Hill, Inc., Singapore.

[5] Gary W. Johnson, 1994. "LabVIEW Graphical Programming". McGraw-Hill, Inc., New York, U.S.A.

[6] J. Michael Jacob, 1989," Industrial Control Electronics". Prentice-Hall International, Inc., New Jersey, U.S.A.

[7] National Instruments, 2002, LabVIEW Basics Introduction, Course Manual, U.S.A.

[8] National Instruments, 1996, "LabVIEW Graphical Programming for Instrumentation". User Manual. New Jersey, U.S.A. 